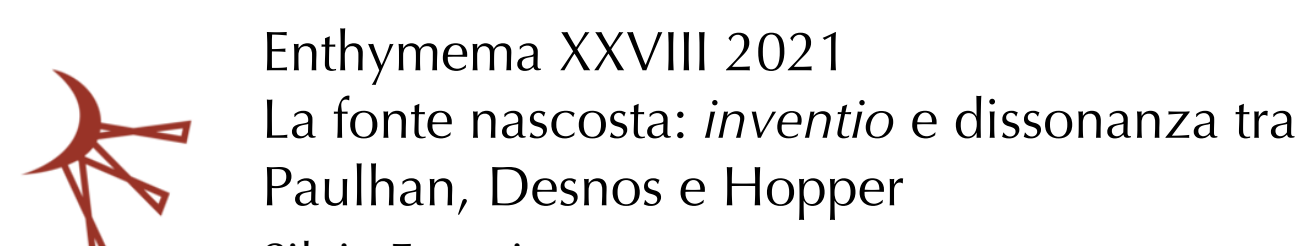

ENOYMHMA Silvia Ferrari

Università del Piemonte Orientale

\begin{abstract}
L'articolo intende partire dal concetto di automatismo insito nel luogo comune, inteso nella sua accezione più ampia, per enucleare alcuni punti fondamentali della riflessione di Jean Paulhan, direttore della NRF negli anni centrali del Novecento, anche alla luce del contributo di Henri Meschonnic in relazione al proverbio in quanto atto linguistico caratterizzato da ri-enunciabilità. Le considerazioni sul ritmo che ne conseguono, già sfruttate dalle sperimentazioni linguistiche di Paulhan e da dadaisti e surrealisti, vanno nella direzione di riscoprire il côté intersoggettivo che, quale fonte nascosta alla base del luogo comune, permette anche di mettere in discussione e rivitalizzare i cliché stessi. Rielaborazione che funziona da specola nei confronti dei pregiudizi e degli automatismi nascosti nel linguaggio. Verranno così evidenziati i punti di contatto tra la fonte nascosta che sostanzia il luogo comune, il défigement operato da Robert Desnos e le tele di Edward Hopper.

Parole chiave - Paulhan; Desnos; Proverbio; Inventio; Défigement.
\end{abstract}

\begin{abstract}
The article begins with the inherent automatism of the use of common sayings in a wider interpretation in order to clarify some of the most fundamental points of reflexion of Jean Paulhan who was director of the NRF in the middle years of the twentieth century. Furthermore, Henri Meschonnic's contribution will be taken into account. The following remarks on rhythm which have already been used by Paulhan as well as Dadaists and surrealists lead us to the rediscovery of the intersubjective aspect which, as hidden sources of the base of common sayings, allow us to discuss and revitalize those clichés. This reworking gives the possibility to observe the prejudices and the hidden automatisms in language. The various points which link the hidden source from which common sayings originate, the défigement belonging to the poet Robert Desnos and the canvasses painted by Edward Hopper, will come to light.
\end{abstract}

Keywords - Paulhan; Desnos; Proverb; Inventio; Défigement.

Ferrari, Silvia. "La fonte nascosta: inventio e dissonanza tra Paulhan, Desnos e Hopper". Enthymema, n. XXVIII, 2021, pp. 145-159.

http://dx.doi.org/10.54103/2037-2426/15877

https://riviste.unimi.it/index.php/enthymema

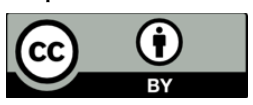

Creative Commons Attribution 4.0 Unported License

ISSN 2037-2426 


\title{
La fonte nascosta: inventio e dissonanza tra Paulhan, Desnos e Hopper
}

\author{
Silvia Ferrari \\ Università del Piemonte Orientale
}

\begin{abstract}
Je suis près de penser que Jean Paulhan n’a jamais écrit que des récits ou toujours sous forme de récit. De là cette gravité qu'il voudrait, par discrétion, nous rendre légère, cette recherche qui ne s'arrête pas et ne s'interrompt que pour se prendre par un mouvement continu (le mouvement de raconter), alors que cette continuité n'est propre qu'à cacher les vides qui ne se laissent pas voir et cependant pourraient laisser passer une lumière ou bien la laisser se perdre, comme il arrive par le phénomène de la transparence.
\end{abstract}

Maurice Blanchot, L'Amitié 173

Citato spesso come l'«eminenza grigia della Nowvelle Revue Française», di cui fu direttore dal 1935 al 1940 e dal 1953 al 1968, Jean Paulhan (1884-1968) si concentra, continuando la riflessione di Paul Valéry, sul nesso, inestricabile, che tiene insieme critica e poetica, inventio ed estetica. Fu apprezzato dai surrealisti per i suoi scritti sui proverbi (da cui questo studio intende partire) e per il romanzo, in parte autobiografico, Le guerrier appliqué (1917). Questo resoconto, ispirandosi all'esperienza vissuta a 30 anni, in un reggimento di zuavi, inquadra, in modo disincantato e scevro di ogni celebrazione retorica, la trasformazione subita al fronte da uomini sottoposti a un progressivo e inesorabile adeguamento all'automatismo innescato dalla cieca esecuzione degli ordini: «Les premières heures de marche nous avaient surpris et fatigués, les suivantes eurent un effet moins simple: dans le même temps que la fatigue, il semblait que se développaient en nous toutes les forces qui s'opposent à la fatigue» (Le guerrier appliqué 19), «Sans que rien encore n'eût été présenté de la guerre, je la ressentais en moi et la trouvais naturelle» (23), «la conscience guerrière qui se forme, évidemment, en moi» (25), «la dignité des animaux me frappait» (29), «je tremble, je n'ai pourtant aucun sentiment qui ressemble à la peur» (36), «Un inconnu, s'il était venu me jeter un ordre à l'improviste, je crois que j'eusse obéi par négligence, avant de songer à en demander la raison» (48). Automatismo in cui si potrebbe già vedere in nuce il nucleo della riflessione, costante, che Paulhan condurrà sul rapporto tra contrainte e ispirazione, tra Rhétoriqueurs e Terroristes, soprattutto se pensiamo che a quel primo libro segue un romanzo dal titolo ancora più significativo, ossimorico, La Guérison sevère (1920), che appare come la prosecuzione dell'azione di spersonalizzazione innescata dalla disciplina militare. Ma è a partire da L'expérience du proverbe (1925) e da Les Fleurs de Tarbes $(1936 / 1941)$ che la riflessione sulla contrainte, linguistica e letteraria, assume un impianto teorico, per quanto mai sistematico. Una critica che, come osserva Luciano Anceschi, «con un gesto leggero contribuisce a definire i confini di un'area morale delle lettere, in cui il gusto della riflessione non rifiuta i piaceri dell'immaginazione, e viceversa» (303). E, continua Anceschi,

Appare subito certo che Paulhan non ama le «soluzioni definitive», ha non so che sospetto per le acquisizioni assolute; e così, nell'esame continuo e minuzioso della sua «scienza della parola», egli preferisce ogni volta seguire lo sfaccettarsi del problema nei suoi molteplici aspetti [...] il Terrore, per esempio; ecco un'immagine, anzi un emblema [...] assai caro a Paulhan, che di esso si giova per indicare e comprendere tutta una regione di realtà letteraria. Ebbene, due sono le 


\section{La fonte nascosta: inventio e dissonanza}

\section{Silvia Ferrari}

condizioni per intendere la direzione di movimenti di siffatta realtà: che il Terrore sia considerato come una situazione perpetua - talora latente, ma sempre pronta a scatenarsi all'improvviso - delle forme letterarie; e che si riconoscano le ragioni storiche - dal romanticismo al surrealismo - del suo particolare avverarsi nel nostro tempo.

Il Terrore, in ogni caso, è (dispiace ridurre a schema ciò che ha ben altra vita nel testo) la letteratura à l'état sawvage, la letteratura che, per un desiderio di innocenza e d'immediatezza, rinunzia alle regole, ai metodi, ai clichés per vivere non so che di libertà e purezza. (305-12)

L'approccio fenomenologico (Demont, "L'approche") applicato alle figure, ai cliché e ai proverbi rovescia spesso l'argomentazione critica in narrazione e viceversa. Un caso emblematico è il testo De la poursuite des images ou Le tailleur chinois (scritto negli anni 1919-1921) in cui Paulhan smaschera il luogo comune che vede il poetico nella ricerca di immagini inedite a tutti i costi.

La métaphore [...] traduit entre les interlocuteurs un défaut d'entente; nous y recevons ce que l'on nous dit à l'envers et sur un plan différent. [...] Nous n'entendons pas les mots directement, mais suivant le sens que nous leur formons. La présence de l'image dans ce sens révèle un retard, une rupture de l'entente - et comme un court-circuit de langage - [...] On a récemment trouvé que les poèmes malgaches exotiques qui nous paraissent au plus haut point riches d'images se trouvent faits, de vrai, d'un entassement de lieux communs, et de proverbes, soit hain-tenys malgaches soit chiking chinois. (Cuvres complètes 136-38)

Per illustrare questa tesi, Paulhan ricorre a una sorta di novella, che vale la pena riportare di seguito per intero:

Le tailleur chinois obtient d'une servante qu'il conserverait quelques jours la robe de la reine, dont il voulait surprendre la coupe. Mais il n'avait pas d'autre endroit où la ranger, elle fut dans sa case aussitôt trouée des mites et des papillons, pour les rares qualités de son étoffe. Dans la suite, le tailleur l'imita patiemment; mais avec le plus de soin il reporta sur la nouvelle robe les divers trous, y voyant quelque secret. Ainsi les poètes chercheurs d'images, se pipant aux mots, prennent pour idéal le même défaut d'où le véritable poète s'évadait à grand-peine. (CEuvres complètes 140)

L'aneddoto illumina la critica, lasciando emergere un senso da svelare, quella fonte nascosta alla base del racconto stesso:

C'est par le mouvement du récit (la discontinuité du récit continu) que nous connaissons peutêtre le mieux Jean Paulhan qui s'en écarte, mais qui ne manque pas aussi de se confier parce qu'il découvre - c'est une des premières découvertes d'écrivain - qu'il suffit de dire les choses pour qu'on ne les croie pas, de les montrer du doigt pour qu'on ne voit plus que le doigt [...] et il n’y a là ni ruse ni perversion, sauf le détour qui est propre à l'écriture-lecture, ce double jeu dont il va chercher désormais à rendre compte en le racontant, non pas seulement pour mieux en jouer, mais pour saisir, en cette duplicité, la trace d'une Vérité une ou la découverte du secret comme secret. (Blanchot 174)

Il perno del suo ragionamento diventa, appunto, la fonte nascosta dell'aneddoto, l'idea generatrice del racconto, in un gioco narrativo di specchi in cui $\mathrm{i}$ fini si confondono con $\mathrm{i}$ mezzi. Infatti, prosegue Blanchot:

Le récit révèle, mais, le révélant, cache un secret: plus exactement, il porte. Ce secret est l'attrait visible-invisible de tout récit, de même qu'il a pour effet de transformer en pur récit les textes qui ne semblent pas appartenir à la pratique narrative. Le secret: simplement quelque 


\section{La fonte nascosta: inventio e dissonanza}

\section{Silvia Ferrari}

chose à trouver, ce qui prête à plusieurs narrations un faux air de document à clé; à la fin, nous avons la clé, un moyen d'ouvrir l'histoire, de récompenser l'attente. (Blanchot 177)

Una pratica di disvelamento, in atto, che non prevede un enunciato-rivelazione:

Mais cherchons plus patiemment. S'il y a un secret, ce secret qui rend possible le cours du récit, c'est que le récit le détient par avance, mais ne le dit pas (au moins directement), porté par lui qui le porte. [...] Ce secret est donc le plus commun (et parce que trop connu, heureusement inconnu): c'est le bien de tous, le lieu commun par lequel, divisés, nous communiquons à notre insu. [...] le langage contient le secret (serait donc au-delà de lui), il faut ajouter: mais le secret est au-delà du langage, s'il est non seulement ce dont le langage toujours parle sans en parler, mais ce qui lui donne la parole, à condition d'être laissé lui-même hors discours. (Blanchot 178)

Il luogo comune diventa quel luogo «geometrico» che permette una proiezione intersoggettiva, funzionando anche da chiave di volta di un'impalcatura narrativa. D'altra parte, proprio per essere sempre sotto i nostri occhi, i luoghi comuni, nel senso più ampio del termine, ci sfuggono e, come osserva Paulhan in Les Fleurs de Tarbes:

Ils ne sont pas communs. Malgré leur nom. Malgré l'apparence. Et bien au contraire, s'il est un trait qui les caractérise - et d'où proviennent, de l'inertie à la confusion, les vices que l'on a vus - c'est qu'ils sont par excellence une expression oscillante et diverse, qui prête à double ou quadruple entente; et comme un monstre de langage et de réflexion. (Les fleurs de Tarbes 148)

I luoghi comuni, per la loro forza comunicativa, danno l'impressione di essere un ibrido tra pensiero e parole, di rappresentare un ponte, senza soluzione di continuità, tra signifié e signifiant. Al punto che, prosegue Paulhan,

L'on devine mille autres usages: l'ironie, l'insistance, la déformation légère, un subtil décalage, une chute de voix, en ménageant autour du cliché comme une zone de réflexion, suffisant à nous avertir que nous "pouvons y aller», que nous ne risquons pas d'être dupes, et que l'auteur et nous sommes bien du même côté du lieu commun. (Les fleurs de Tarbes 150-51)

Il luogo comune, dunque, che sia pronunciato con la solennità che di solito lo accompagna o che sia, invece, sottoposto a trattamento ironico, produce, in ogni caso, un effetto di connivenza tra gli interlocutori in grado di riconoscerlo.

Molteplici e mai esaurienti sono le definizioni di luogo comune e di proverbio che si sono succedute nel tempo: talvolta si è posto l'accento sull'autorità popolare di una sentenza, talaltra sulla brevità e sullo schema ritmico. Proponiamo qui la definizione di Henri Meschonnic, che ha il merito di mettere in risalto il côté intersoggettivo che abbiamo già colto attraverso la riflessione di Paulhan:

Le proverbe, comme en cela le poème, est une activité de langage, un acte de discours dont le référent est l'énonciateur et le ré-énonciateur dans leur rapport à une situation. L'énonciation comme construction de la ré-énonciation est le référent. [...] C’est pourquoi la sagesse ne suffit pas à décrire ni ce qu'est ni ce que fait un proverbe. Un proverbe s'inscrit dans une situation, et il inscrit en lui, par la fixité de sa formule, cette situation, - ce qui le spécifie par rapport à d'autres activités de discours qui sont seulement inscrites dans leur situation. Il y a donc dans l'énonciation d'un proverbe un faire particulier, dont les joutes (comme dans les hain-tenys étudiés par Paulhan) réalisent une dénudation expérimentale du procédé. D’où, aussi, de nouveaux proverbes. Dada et les surréalistes ont fait des anti-proverbes. (Meschonnic 426)

Il proverbio interviene quindi come una voce fuori campo in grado di commentare una situazione, riconducendola a una certa categoria dell'esistente: «comme l'énigme et le mythe, 


\section{La fonte nascosta: inventio e dissonanza}

\section{Silvia Ferrari}

le proverbe serait une tentative empirique de mettre le monde en ordre. Ce que montrent les proverbes numériques dans la Bible» (Meschonnic 421). Ma, a dispetto delle apparenze e dei luoghi comuni, non si tratta di un ordine univoco e stabilito una volta per tutte:

Le «style proverbe» n'est donc pas la «voix de la sagesse traditionnelle». Le mode de réénonciation caractérise le proverbe : pas une mentalité, elles y sont toutes. Sa ré-énonciabilité est une signifiance, émission de sens par l'organisation de signifiants. Prescriptif ou descriptif, un proverbe fixe un hors-langage par une motivation mutuelle (rythmique, prosodique, syntaxique, lexicale) des éléments du langage. Tous les éléments jouent ici un même rôle, dans leurs types divers, en séries analogiques. Ce n'est pas l'allitération, le couplage ou la paronomase qui font le proverbe, mais la domination du dit sur une situation à dire qui mobilise le langage. (Meschonnic 428)

Nulla di scontato, quindi, nel luogo comune denunciato da un proverbio, anzi, proprio la ricchezza molteplice e contraddittoria del reale che vi si può manifestare conferisce al proverbio non solo la sua forza persuasiva, ma anche le sue potenzialità creative in termini di inventio. Infatti, solo in un primo momento della disamina critica le posizioni dei Terroristes e dei Rhétoriquers appaiono nettamente contrapposte:

Le réalisme et le surréalisme sont ici logés à la même enseigne. Tous deux mettent en code un curieux système d'alibi. Simplement l'écrivain s'efface ici devant le document humain, là devant le document surhumain. La tranche de vie comme la tranche de rêve lui permettent également de dire «je n’étais pas là». [...] Une dame se promenait avec une rose. Le garde lui a dit: «Vous savez bien qu'il est défendu de cueillir des fleurs. - Je l'avait en entrant, a répondu la dame. Eh bien, il sera défendu d'entrer avec des fleurs». (Les fleurs de Tarbes 38-9)

Il ricorso ai fiori della retorica tradizionale sembra oggetto d'ostracismo da ambo le parti: «il pèse sur l'écrivain de clichés un reproche de paresse ou de facilité» (Les fleurs de Tarbes 445).Ma l'intenzione più subdola della Terreur viene smascherata: «ce que veut la Terreur n'est pas tant que l'écrivain soit inventeur, différent, unique - c'est qu'il s'exprime et se fasse entendre malgré sa différence. Or le succès de l'expression sera difficile - et plus méritoire - à proportion que l'auteur est plus singulien» (Les fleurs de Tarbes 162). Riabilitato dunque il cliché, il proverbio, il luogo comune in generale, resta da capire come la sua fonte nascosta possa risultare foriera di ulteriore rinnovamento e del proverbio stesso e della lingua che lo accoglie:

Or, tout ce que nous avons découvert est que le cliché exige - pour ne point devenir le signe d'une défaite, et d'une lâcheté - d'être sans cesse considéré, remis en question, nettoyé. Comme s'il fallait répondre par un excès de langage, à cet excès de sens: par un excès de matière, à cet excès d'esprit. (Les fleurs de Tarbes 172)

L'eterno rinnovamento del cliché è da Paulhan chiarito con un parallelo con quanto avviene nell'organismo umano: «et l'homme non plus qui ne s'est jamais exercé n'est pas plus libre de corps (si même il se rêve âme pure) que celui qui s'entraîne et se rend maitre de ses actes. Mais tout au contraire assez étroitement tenu par ce corps machinal et comme stéréotypé» (Les fleurs de Tarbes 172). Soltanto il confronto costante e la rimessa in discussione dei luoghi comuni può ripagare l'arte del tributo che essa stessa offre allo scorrere inesorabile del tempo: «Séduit par le potentiel énigmatique des proverbes malgaches, Jean Paulhan pose les jalons d'une conception de l'aphorisme (maximes et condensés de sagesse populaire mais aussi locutions idiomatiques et phrases figées) dont l'écho se prolonge chez les surréalistes». (Angeli 280) 


\section{La fonte nascosta: inventio e dissonanza}

\section{Silvia Ferrari}

L'«apparente innocence du proverbe et sa souterraine énergie dévastatrice» (Angeli 282) sono sfruttate, in particolare, dall'arte combinatoria di Michel Leiris, Paul Éluard, Benjamin Péret et Robert Desnos. In questo studio prenderemo in considerazione le sperimentazioni di quest'ultimo. Sin dagli aforismi di Rrose Sélavy (alter ego del poeta, preso a prestito dallo pseudonimo dell'artista Marcel Duchamp e dal significato «Eros è la vita»), l'autore si misura con la sfida di forzare i limiti della lingua. Appassionato di alchimia e di folklore, sottopone a un processo di anarchico sregolamento e ibridazione generi, cliché e proverbi. È il caso, ad esempio, del romanzo onirico La liberté ou l'amour! (1927) che mescola l'esotismo del romanzo d'avventura con il resoconto di un giro turistico nella ville lumière. Kiyoko Ishikawa osserva, in Paris dans quatre textes narratifs du surréalisme. Aragon, Breton, Desnos, Soupault (1999), che il testo di Desnos è quello più metaletterario: « chez Desnos, Paris n'est point descriptif; c'est un dépôt de signes - noms de rues, de monuments, de places, affiches, statues - destinés à transformer sur le plan linguistique et sémantique» (Ishikawa 158). Il titolo stesso è un calembour paronimico del grido dei rivoluzionari: «la liberté ou la mort!» (Ishikawa 168). Ma come denuncia anche la coeva riflessione del filosofo Walter Benjamin sull'usura del linguaggio, quello che interessa soprattutto ai surrealisti non è tanto l'attacco alla lingua e alla letteratura in sé quanto l'attacco alle convenzioni e alla spietata macchina del progresso innescata dal capitalismo:

Comme beaucoup d'autres surréalistes, Desnos voit parfois dans Paris des ruines de la civilisation du temps industriel; pour lui, c'est une ville fantôme qui a autrefois été très prospère. Le début de Denil pour denil, texte narratif écrit juste avant La liberté ou l'amour!, nous offre un paysage apocalyptique des ruines de Paris : «Ces ruines sont situées sur les bords d'un fleuve sinueux. [...] Au sud-ouest s'élève une construction métallique ajourée, très haute et dont nous n'avons pu déterminer l'usage. Elle paraît prête à s'écrouler car elle penche fort et surplombe le fleuve». (Ishikawa 180)

Di qui si vede come la condanna di Paulhan del surrealismo, che pure appare ritratto tra Paul Éluard e Banjamin Péret nel quadro di Max Ernst Au rendez-vous desamis (1922) (Demont, "Le dadaïsme et le surréalisme" 28), tenda, in realtà, ad assimilare surrealismo e dadaismo sotto l'accusa di «misologie». Quello che comunque sconcerta il pensatore, riguardo ai proclami di Breton, è il fatto che «la littérature et l'écriture lui apparaissent non comme une fin en soi, mais comme des outils devant libérer l'homme» (Demont, "Le dadaïsme et le surréalisme" 29). Non è un caso se la versione di Les Fleurs de Tarbes del 1941 delinea più chiaramente i suoi attacchi al surrealismo e a Breton di quanto non facesse l'edizione del 1936 (Demont, "Le dadaïsme et le surréalisme" 26). Ad allertare Paulhan è, d'altra parte, la situazione determinata dalla svolta del 1885 nell'istruzione nazionale:

Alors que, depuis 1885, le recul de la rhétorique dans l'enseignement secondaire est net, les classes de rhétorique changent de nom en 1902, suite à un décret entre autres signé par Gustave Lanson. [...] On étudie alors les œuvres complètes de "grands auteurs» dont la lecture paraît socialement et moralement utile. (Demont, "Le dadaïsme et le surréalisme" 23)

Una situazione di crisi che Paulhan vede amplificata dal tipo di critica di cui è, consapevolmente o meno, portatrice:

Démonte adroitement son auteur, et le [met] devant nous en petites pièces: vices et vertus, amourettes, coucheries, lectures, relations fâcheuses, voisins d'étage. Psychanalyste, il remonte jusqu'aux chagrins et plaisirs de la vie utérine, masturbations, amours incestueuses et le reste. Il décèle adroitement sous le poème ou le récit la ruse de classe, la machine à vapeur, la puissance des trusts. (Paulhan, Petite préface à toute critique [1951] cit. in Demont, "Le dadaïsme et le surréalisme" 24) 


\title{
La fonte nascosta: inventio e dissonanza
}

\section{Silvia Ferrari}

La Terreur, con la sua smania di novità e di individuo, con il pretesto dell'autenticità, sembra fagocitare la specificità stessa del fatto letterario.

Paulhan voudrait donc endiguer l'évolution de la Terreur, qui attaque, en plus de la littérature, les fondements du langage commun, ce qui revient, selon une idée d'époque, à vouloir saper la base même du système démocratique [...] car il sait bien que le langage implique répétition, reconnaissance et confiance, puisque chaque langue soutient une vision du monde partagée. (Demont, "Le dadaïsme et le surréalisme" 33-5)

Ma l'intento eversivo con cui i surrealisti, e in particolare Desnos, manipolano il linguaggio va proprio nella direzione di rivitalizzare e recuperare alla lingua quei «relitti» linguistici che si presentano ai loro occhi come le rovine della società borghese, accanto a merci che, prodotte in serie, diventano obsolete quasi ancor prima di uscire dalla catena di montaggio. Questa visione filmica e barocca della crisi tra le due guerre, che prosegue e si esaspera durante gli anni del secondo conflitto mondiale, prende le forme di un apparente non-sense linguistico che scardina luoghi comuni e miti nazionali. Non è un caso se negli anni Venti la polizia parigina vieta di arrangiare la Marsigliese in jazz (Marmande 303), mentre spopolano le parodie della Marsigliese stessa e del Padre nostro:

La parodie surréaliste transforme parfois une seule substitution des expressions appartenant sans doute à tout le monde mais que l'accaparement bourgeois a raplaties en clichés mensongers. Cette opération ressemble quelque peu à l'astuce de Man Ray attachant des clous à un fer à repasser et le nommant cadeau. (Riese 116)

Alcuni testi appaiono significativi in tal senso, in quanto permettono di cogliere le potenzialità creative, dal punto di vista dell'inventio, del trattamento surrealista del luogo comune, sempre inteso in senso lato, in varie tappe dell'opera di Desnos. In più occasioni l'autore rivitalizza l'antica metafora del miele-poesia, sin dalla raccolta Prospectus (1919), dove irride agli slogan del sano ed efficiente mondo borghese:

\section{A Paul Smara}

\author{
Elles sont mortes les abeilles \\ au cimetière des Lilas ${ }^{1}$ \\ Si vous voulez du chocolat \\ Mettez deux sous dans l'appareil \\ Il est mort notre Apollinaire \\ et mort aussi Laurent Tailhade \\ Cinq abeilles volent dans l'air \\ et les sirènes de naguère \\ pour moi s'abattent dans la rade \\ Meurent les porte-lyre \\ le rimeur Jean Aicard \\ ouvre la bouche en tirelire
}

\section{SI VOUS VOULEZ DU CHOCOLAT} METTTEZ DEUX SOUS DANS L'APPAREIL.

5.11.19. (Cuvres 22-3)

${ }^{1}$ Probabile riferimento alla storica Closerie des Lilas, oggi noto ristorante di Montparnasse, luogo di ritrovo di intellettuali tra XIX e XX secolo. 


\section{La fonte nascosta: inventio e dissonanza}

\section{Silvia Ferrari}

Morti i poeti modello per i surrealisti, come Apollinaire e lo sferzante Tailhade, restano i rimatori a comando, quelli votati a fabbricare testi commerciali, come Jean Aicard, che «apre la bocca a salvadanaio», in un calembour che contrappone il neologismo composto "portelyre», a designare i veri scrittori, e quelli che riempiono solo la loro «tirelire», il salvadanaio. Ma lo slogan riportato nei distributori automatici contiene una palese e veemente critica alla società meccanizzata. La situazione evocata ci fa venire in mente il quadro di Edward Hopper Automat (1927), dove il titolo allude sicuramente al bar-ristorante automatico in cui si trova la ragazza rappresentata, ma per l'atteggiamento della stessa, scivola come definizione anche sulla figura umana che, nella fretta dei ritmi frenetici della città, consuma il suo caffè dopo aver tolto un solo guanto (Sounac 443).

Le titre de l'œuvre vise à provoquer un court-circuit sémantique. «Automat» est le terme générique qui désigne un nouveau type de restaurant apparut à New York en 1912 (le premier restaurant de ce genre fut créé à Berlin, puis transposé aux États-Unis en 1902 à Philadelphie). On y sert une nourriture délivrée par des distributeurs automatiques. Hopper se saisit de ce symbole de la mécanisation des sociétés modernes. Il s'en empare pour l'occulter aussitôt, préférant vouer son image aux effets déshumanisants qu'il produit. Ne subsiste plus qu'une figure, dont le geste et le mutisme ont rejoint ceux de la machine dont le peintre lui attribue indirectement le nom. (Ottinger 58-9)

Sia il componimento di Desnos sia l'opera di Hopper ci calano in uno strano tempo acronico, quello che Katharine Conley ha definito «an immobilized present», «baroque time» (Conley 34). Un tempo sospeso, una scheggia di eternità: «Les tableaux de Hopper ont été lus comme autant de fotogrammes, d'instants figés, vitrifiés à l'intérieur d'un récit, comme les instantanés d'une scène interrompue» (Ottinger 15). Orietta Rossi Pinelli attribuisce alla frequentazione delle tele di Vermeer, «osservate dal vero sia a New York che ad Amsterdam [...] l'esplicita intenzione di sottrarsi alla definizione della temporalità come movimento, come agente di trasformazione» (Rossi Pinelli 19).

Possiamo leggere nella récursivité irriverente e dissacrante degli scritti di Desnos come nella denuncia silenziosa di Automat, un monito che ha la stessa solennità atemporale di un proverbio, di un cliché, di un luogo comune e che ci rinvia a una situazione generica, la quale, a sua volta, può prefigurare una particolare circostanza. Questo è il potenziale dell'inventio narrativa insita in questi dispositivi che funzionano come enunciazioni in atto, e non come enunciati. Un potenziale d'inventio ben ravvisato da Lawrence Block, che così apre un'antologia di racconti ispirati a quadri di Hopper:

I suoi quadri non raccontano storie. Ma hanno la capacità di evocare in modo potente e irresistibile quelle racchiuse al loro interno in attesa di essere raccontate. Hopper sa fermare sulla tela un momento sospeso nel tempo - un istante con un passato e un futuro che lo spettatore è chiamato a rintracciare. (Block VI)

Condotto sul filo dell'ironia, il racconto ispirato a Automat dà consistenza ai dettagli del quadro: «era il cappellino a fare la differenza», «del resto quella era una tavola calda con distributori automatici di cibo, e la povera ragazza era quanto di più simile a un automa si potesse immaginare», «in fondo era proprio così: per quanto trasandati, gli abiti che aveva addosso la qualificavano in modo inequivocabile come una vera signora» (Block 275-293). I personaggi e i paesaggi dei suoi quadri hanno la genericità del luogo comune: «dai suoi disegni preparatori appare evidente che sono stati cancellati i segni che avrebbero fatto del modello una persona particolare» (Bonnefoy 53). Dall'aneddoto in cui riconosciamo, senza che sia esibito, l'imperativo del consumismo, passiamo a un'altra tela che nasconde, di nuovo con una sapiente orchestrazione, un cliché ancora legato alla società dei consumi. In Chop Suey 


\section{La fonte nascosta: inventio e dissonanza}

\section{Silvia Ferrari}

(1929) due amiche sono ritratte in un locale, ma la malizia con cui appare tagliata l'insegna del locale e i colori accesi fanno scadere la scena amicale in un voyeurismo da bordello, dove $\mathrm{i}$ corpi anonimi delle due giovani sono ridotti al rango di merce. La donna quale allegoria della merce in vendita nel mondo capitalista:

Le finestre del dipinto possono essere paragonate a zone di confine atte a mettere in risalto l'interno del locale. Superfici geometriche colorate compongono la finestra sullo sfondo ed è difficile capire se si tratti del muro di una casa situata di fronte o di una parte di cielo riflessa sul vetro della finestra. La finestra sulla destra concede senza dubbio una vista molto limitata: una facciata, una scala antincendio, un pezzo di cielo e l'insegna luminosa del Chop Suey. Le donne con i loro copricapi liberty e i loro visi molto truccati assomigliano a bambole immobili. [...] I visi delle donne truccate danno, tuttavia, un'idea di seduzione. L'insegna del locale Chop Suey fa venire in mente un quartiere di piaceri, il rosso abbagliante del cartello richiama le labbra truccate delle signore e le lettere non completamente visibili della parola «Suey» fanno sì che sia possibile associarvi, come in un rebus, la parola «Sex». I segni della seduzione, della solitudine e dell'immobilità si intersecano l'uno nell'altro; come altri dipinti di Hopper anche questo permette e richiede una doppia lettura. (Renner 69-70)

Ma dagli studi sui proverbi malgasci Paulhan e i surrealisti avevano senz'altro individuato una caratteristica propria del proverbio: la forza insita nel sostrato comune che permette a ogni locutore di una determinata civilisation di riconoscervisi o, comunque, di riconoscerlo, è in grado di allertare l'attenzione e, addirittura, di porre fine a controversie. Così l'autore annota in L'expérience du proverbe la sua esperienza in Madagascar:

La richesse de mon vocabulaire n'était pas ici en cause, ni la correction de ma syntaxe [...] il me semblait plutôt qu'il manquait à mes paroles un certain poids, une valeur, un ton de conviction. [...] Je n'ai jamais vu personne interrompre les proverbes, mais il semblait au contraire que chacun portât son attention à les favoriser, à les faciliter - comme l'on est « de cœur » avec un acrobate qui accomplit un tour dangereux. (CEuvres complètes 101-2)

Paulhan si rende conto del valore di cooperazione, di coesione garantito dal proverbio, ma, al contempo, coglie quello che, come evidenziato sopra con Meschonnic, caratterizza il discorso in atto del proverbio: la sua appropriatezza a una determinata situazione.

Il semble qu'il y ait à l'intérieur du proverbe quelque difficulté, quelque nœud qui exige, pour être saisi, que l'on considère d'abord ce proverbe dans son application et dans son jeu. [...] Tout se passait comme si ce proverbe mal employé et contraint d'avouer sa nature de proverbe venait en aide à l'opinion qu'il attaquait plutôt qu'à celle qu'il devait soutenir. (CEurres complètes 108-10)

Di qui il passaggio a considerare, ancora una volta, quanto la forza dell'immagine si dilegui in un pallido riflesso del concetto: «le proverbe est à la fois moins et plus qu'un raisonnement ou une métaphore : il est l'un et l'autre à l'état figé» (CEuvres complètes 111). E questo indipendentemente dal tasso, per così dire, immaginifico del proverbio, se consideriamo, con Meschonnic, che: «les proverbes à métaphore et les proverbes sans métaphore ont un même fonctionnement: c'est le fonctionnement qui fait la figure, pas l'énoncé. On le place, et replace. Ils présupposent et installent le dialogique, dans le monologue comme dans la narration» (Meschonnic 427). Per questo Meschonnic condensa il suo ragionamento in una formula emblematica: «ce n'est pas comme métaphore qu'un proverbe est un proverbe, c'est comme proverbe qu'un proverbe est métaphore» (Meschonnic 427).

L'argomentazione di Paulhan prosegue, individuando nel proverbio un elemento del discorso che può essere considerato a sé, quasi si trattasse di una parola sola: «C'est la phrase 


\section{La fonte nascosta: inventio e dissonanza}

\section{Silvia Ferrari}

proverbiale entière que je devais me rappeler, comme si elle n'eût été qu'un seul mot. [...] Il arrivait par la suite que le cadre abstrait, l'armature commune à toute une famille de proverbes se présentât d'abord à mon esprit: ce cadre ensuite se garnissait de mots» (Euvres complete 111-2). L'individuazione di un moule, di uno stampo in grado di classificare, ma anche riprodurre, famiglie di proverbi caratterizzati da strutture morfosintattiche analoghe apre la via alla creatività surrealista e fa approdare Paulhan alla seguente considerazione: «Or, j’avais pris pour des mots ce que les Malgaches entendaient en choses. [...] Certains mots doivent être tenus pour choses. [...] ils sont encore des choses singulières, qu'il est urgent de dire, et de dire le plus exactement possible» (CEuvres complètes 123-24). In questa esattezza sia Meschonnic sia Paulhan riconoscono l'importanza del ritmo: «Il faut d'abord reconnaitre au rythme, à la rime et aux pieds cette valeur et ce mérite singulier: c'est qu'en rendant à l'esprit les stéréotypes et les lieux dont notre attention le privait, ils le restituent à son état premien) (Euvres complètes 183).

Anche ricorrendo all'espediente del ritmo, Desnos cerca di intaccare il paludato perbenismo borghese, come denuncia la seguente poesia, in cui un proverbio viene stravolto a sottolineare l'opera di dissonante engagement portata avanti dalla sua poetica:

\section{HISTOIRE D’UNE ABEILLE}

Abeille bruissante des matins d'été,

Abeille qui bourdonnes dans la tasse,

Abeille où es-tu allé ?

Abeille bruissante et jamais lasse.

J'ai construit ma ruche

Dans la cervelle d'un enfant ${ }^{2}$

Mais tant va l'abeille à la cruche ${ }^{3}$

Que la fleur fleurit dedans.

Ce furent d'abord les yeux étonnés

Et le miel, et la cire bien construite,

Le sourire et le rire et le mot chantonné

Et la question jamais détruite.

Tant qu'à force de bourdonner

Dans la cervelle de l'enfant

Il finit par s'en étonner

Et par inquiéter ses parents.

Quand il fut approvisionné

De miel et de cire bien mûrs

Alors je l'ai abandonné

Dans le baiser d'une piqûre.

Mais nul jamais ne fera sortir de sa mémoire

Mon bourdonnement à moi, l'abeille,

Et jamais il ne voudra croire

Aux mots pourris qu'on glisse dans l'oreille,

2 Il verso allude al titolo del quadro di Giorgio de Chirico Il cervello del bambino (1914), opera fondamentale per la cerchia dei surrealisti.

${ }^{3}$ I vv. 3-4 riprendono e modificano il proverbio «Tant va la cruche à l'eau qu'à la fin elle se casse», analogo all'italiano «Tanto va la gatta al lardo che ci lascia lo zampino». 


\section{La fonte nascosta: inventio e dissonanza}

\section{Silvia Ferrari}

Qu'on glisse sournoisement

Dans l'oreille des enfants,

Avec la complicité des parents.

1936. (CEuvres 986-7)

A parte la ripresa, sopra menzionata, della metafora classica miele-poesia e la citazione del quadro simbolo del periodo dei sonni ipnotici Il cervello del bambino di De Chirico (1914), che rappresenta un uomo con gli occhi chiusi davanti all'edizione francese di Così parlò Zarathustra di Nietzsche, questo testo, mediante l'espediente del dialogismo, a partire dalla seconda quartina fa prendere la parola all'ape che rappresenta l'allegoria dello stesso Desnos. È il poeta, plasmando la cera in costruzioni perfette, a instillare il dubbio nella mente del bambino, rendendolo immune ai pregiudizi inculcati in modo subdolo dai genitori. Si tratta di una poetica che dispone al sorriso e a un atteggiamento curioso verso la vita, di una poetica sempre disposta a esaltare le cose belle della vita, come testimonierà l'autore fino all'ultimo, fino all'ultima poesia scritta nel campo di smistamento di Compiègne, "Printemps", nell'aprile del 1945, dopo essere stato arrestato dalla Gestapo il 22 febbraio 1944 a seguito di una delazione per aver schiaffeggiato pubblicamente l'antisemita Laubreaux et per aver collaborato attivamente alla rete clandestina Agir. In "Histoire d'une abeille" il proverbio modificato è «Tant va la cruche à l'eau qu'à la fin elle se casse», equivalente al nostro «Tanto va la gatta al lardo che ci lascia lo zampino»: qui Desnos ha completamente stravolto il senso del detto popolare, in quanto «Tant va l'abeille à la cruche que la fleur aleurite dedans» presuppone un fruttuoso andirivieni dell'ape che quindi sortirebbe, appunto, gli effetti auspicati dalla poesia di Desnos sullo spirito critico del bambino.

Un altro proverbio che viene modificato da Desnos appare nel film L'étoile de mer di Man Ray, girato a partire da un poema di Desnos e rappresentato per la prima volta nel 1928. Il proverbio «Il faut battre le fer quand il est chaud» appare trasformato in un intertitre come «Il faut battre les morts quand ils sont froids» (Aurouet 75), che suona come un'irrisione alla celebrazione del cogliere l'attimo propizio per avere successo, perfetto mantra dell'etica capitalista.

Nella seguente poesia, "Faire part", della raccolta C'est les bottes de 7 lieues cette phrase «je me vois» (1926, con acqueforti di André Masson), viene ripreso e stravolto il proverbio «Une hirondelle ne fait pas le printemps», ovvero «Una rondine non fa primavera», in «La dernière hirondelle fait l'automne», ma, come abbiamo evidenziato in nota, i giochi sulle espressioni idiomatiche e sulla polisemia di lessemi come «facteur», qui da intendere sia nell'accezione di «postino» sia in quella di «fattore (di sottrazione)», sono molteplici.

\section{FAIRE PART}

Sur le pont du navire la couturière fait le point ${ }^{4}$ couturière taille-moi un grand paon de mercure ${ }^{5}$ je fais ce soir ma dernière communion ${ }^{6}$

La dernière hirondelle fait l'automne ${ }^{7}$

\footnotetext{
4 Paronomasia in absentia tra due espressioni idiomatiche: quella espressa, fait le point ("fa il punto [della situazione]"), e quella richiamata dal contesto, fait un point (“dà un punto [con l'ago]").

${ }^{5}$ L'espressione ricorrente «un pan de tissu» gioca qui con gli homonymes pan/paon, ovvero parole che, pur avendo la stessa pronuncia ['pã], hanno significato diverso (Dumas 354).

6 «La première communion» diventa «la dernière communion».
} 


\section{La fonte nascosta: inventio e dissonanza}

\section{Silvia Ferrari}

D'entre les becs de gaz blêmes

Se lève une figure sans signification.

Statues de verre flacon simulacre de l'amour

Vient la fameuse dame

Facteur de soustraction ${ }^{8}$

avec une lettre pour moi

Mon cher Desnos mon cher Desnos

Je vous donne rendez-vous

dans quelques jours

On vous préviendra

Vous mettrez votre habit d'outre monde. ${ }^{9}$ (CEuvres 291)

Anche il titolo di un libro di Desnos, cronaca romanzata della sua liaison con la cantante Yvonne Georges, intesa a mettere in guardia contro il pericolo rappresentato dalle droghe, è un proverbio troncato: Le Vin est tiré (1943). Si tratta, infatti, del proverbio «Quand le vin est tiré, il faut le boire», che allude alla necessità di assumersi le responsabilità delle proprie azioni e di subirne le estreme conseguenze. La préface è molto eloquente e colpisce per la sua lucidità quasi preveggente:

Le présent livre essaie, sans que l'auteur croie y être clairement parvenu, de prouver que la question sociale est responsable de la diffusion chaque jour plus grande des drogues [...] poisons qui, ne l'oublions pas, ne sont plus le privilège des classes riches ou intellectuelles mais menacent le prolétariat et toute la nation. Tant que l'ordre social continuera à brimer le libre développement de l'individu, des hommes et des femmes chercheront dans l'opium et l'héroïne d'illusoires compensations et la clef d'un suicide lent. Dans vingt ans la drogue se sera répandue dans tous les milieux, peut-être même dans les campagnes, et il sera trop tard pour remporter la victoire sur elle. (Euvres 1001)

La pratica del défigement da parte di Desnos si applica non solo ai proverbi, ma anche alle frequenti dittologie sinonimiche, alle espressioni idiomatiche, alle polirematiche, creando geometrie linguistiche che ricordano i virtuosismi dell'art déco.

\section{L'OISEAU MÉCANIQUE}

L'oiseau tête brûlée

Qui chantait la nuit

Qui réveillait l'enfant

Qui perdait ses plumes dans l'encrier

L'oiseau pattes de 7 lieues

Qui cassait les assiettes

Qui dévastait les chapeaux

Qui revenait de Suresnes

L'oiseau l'oiseau mécanique

A perdu sa clef

${ }^{7}$ Cfr. supra: il proverbio «Une hirondelle ne fait pas le printemps» si muta in «la dernière hirondelle fait l'automne».

${ }^{8}$ Cfr. supra: il termine «facteur» viene qui usato in senso ambiguo, conservando il duplice significato di postino e di elemento di un'operazione matematica.

${ }^{9}$ La parola «outre-tombe» è cambiata, in modo assai significativo in rapporto alla sensibilità surrealista, nel neologismo «outre-monde». 


\section{La fonte nascosta: inventio e dissonanza}

\section{Silvia Ferrari}

Sa clef des champs

Sa clef de voûte

Voilà pourquoi il ne chante plus. (CEuvres 662)

In "L'oiseau mécanique" il ritmo estrae dalle espressioni idiomatiche «(prendre la)clef des champs» e «clef de voûte» il denominatore comune «clef» che anticipa la pointe finale in cui il lessema acquista un valore musicale. Il soggetto del poema appare in consonanza con La clef des champs (1936) di René Magritte (il tema illusionistico del quadro nel quadro) e con il quadro di Paul Klee Die Zwitscher Machine (La Machine à gazouillis, 1922, esposto al MoMa de New York et classificato nel 1933 tra le opere d'arte degenerata). Quest'ultimo, come il breve poema-cantilena di Desnos, nascondono una critica alla società meccanizzata:

La giration que l'on imagine grinçante du tournebroche-perchoir accompagne les cris perçants de volatiles aux langues acérées, pareils à des leurres métalliques: le caractère suraigu des formes caricaturales traduit le déchirement acoustique. En fait tout le dispositif relaie et transfère l'allitération du titre dans la dimension de la forme, l'audible dans le visible. (Frontisi 230)

Nella poesia "La créole" Desnos mette in scena una Venere nera con un corteggio di angioletti che, infrangendo un ennesimo cliché, descrive neri come il carbone.

\section{LA CRÉOLE}

\section{$[\ldots]$}

Comme une Vénus inconnue

Surgissant de la conque blanche du lit

Elle reposera son beau corps

En rêvant d'un Olympe noir comme elle

Et des anges noirs comme du charbon soulevant cette déesse de couleur l'emporteront vers un pays de ténèbres où brille un soleil éclatant et bleu [...]. (CEuvres 662)

Abbiamo citato sopra l'ultima poesia scritta da Desnos, "Printemps": quella poesia si apre con la menzione di Rrose Sélavy, l'alter ego femminile del poeta negli anni Venti, preso in prestito dall'artista Marcel Duchamp. La prima quartina del sonetto fa entrare l'aria della primavera nella prigionia del poeta:

\section{PRINTEMPS}

Tu, Rrose Sélavy, hors de ces bornes erres

Dans un printemps en proie aux sueurs de l'amour,

Aux parfums de la rose éclose aux murs des tours,

A la fermentation des eaux et de la terre

[...]. (CEvres 1259)

Segno che l'autore ritorna ad un altro 'luogo comune', Pegaso allegoria della poesia, in grado di trasportare lontano, come già scriveva negli aforismi di Rrose Sélavy:

L'orgueil de Rrose Sélavy sait s'évader du cercle qui peut se clore comme un cercueil. (CEurres 148) 


\section{La fonte nascosta: inventio e dissonanza}

\section{Silvia Ferrari}

Rrose Sélavy peut revêtir la bure du bagne, elle a une monture qui franchit les montagnes. (CEurres 503)

Aforisma ripreso nella lettera che Desnos scrive alla compagna Youki dal campo di Flöha, il 7 gennaio 1945, pochi mesi prima della «marche de la mort» che condurrà il poeta a morire a Terezin: «Je trouve un abri dans la poésie. Elle est réellement le cheval qui court audessus des montagnes dont Rrose Sélavy parle dans un de ses poèmes et qui, pour moi, se justifie mot pour mot» (Egger 1047).

Curiosamente scorgiamo il cavallo alato anche in un quadro di Hopper, Gas (1940), dove appare nell'insegna di una pompa di benzina al limitare di un bosco. Anche in questo caso il dettaglio connota in senso lirico una scena prosaica: al crepuscolo, la stazione di servizio presenta delle finestre che irradiano luce da una fonte nascosta, mentre tre pompe di benzina raffigurate in prospettiva, dilatano in profondità lo spazio. Ancora una volta, la chiave del dipinto non è esibita, non è «narrata». Ulteriore maestria di Hopper documenta il quadro Bridle Path (1939): si tratta di un'azione molto dinamica in cui tre cavalieri (due donne e un uomo) vanno al galoppo a Central Park, ma un cavallo sembra imbizzarrirsi all'ingresso di un tunnel. La straordinarietà dell'opera sta nel fatto che l'oggetto che terrorizza l'animale non è mostrato: la fonte dell'azione è nascosta, eppure lo spettatore riesce a riconoscere nel quadro un duplice livello di lettura. Anche nel cuore della città, anche dove l'uomo ha più antropizzato il paesaggio, qualcosa sfugge, la natura può sopraffare il suo dominatore. Ancora più significativo, in tal senso, è Cape Cod Morning (1950), dove una donna di profilo scruta assorta l'orizzonte, protesa in avanti, il corpo arcuato verso il punto d'osservazione che rimane celato allo spettatore. Come si rileva dalla Notice ${ }^{10}$ della sala in cui era esposto il quadro alla Fondazione Beyeler per la mostra Hopper (26 gennaio - 20 settembre 2020) a Riehen (Basilea), «cette focalisation sur ce qui est invisible au spectateur est typique des figures humaines de Hopper».

In conclusione, la critica alla società benpensante e meccanizzata del Novecento, attraverso i suoi cliché, offre sovente una chiave di lettura che, forse troppo spesso ignorata, avrebbe potuto cambiare il corso della storia e, a dispetto della Terreur, abbiamo potuto constatare come quella chiave di lettura sia spesso una fonte nascosta che, grazie alle potenzialità creative del luogo comune, permette a un frammento di eternità di stagliarsi in una dimensione narrativa.

\section{Bibliografia}

Anceschi, Luciano. "Paulhan o dell'ambiguità delle lettere." Aut aut, vol. 10, 1952, pp. 298316.

Angeli, Giovanna. "Surréalisme et proverbe: à la limite du jeu." Jeu surréaliste et humour noir, a cura di Jacqueline Chénieux-Gendron e Marie-Claire Dumas, Lachenal \& Ritter, 1993, pp. 181-300.

Aurouet, Carole. L'étoile de mer. Poème de Robert Desnos tel que l'a vu Man Ray. Gremese, 2018.

Blanchot, Maurice. L'Amitié. Gallimard, 1971.

Block, Lawrence, ed. In Sunlight or in Shadow. Stories inspired by the Paintings of Edward Hopper. Pegasus Book, 2016.

10 A cura di Tasnim Baghdadi, Iris Brugger, Daniel Kramer, Ulf Küster, Katharina Rüppell, Janine Schmutz; traduzione in francese di Maud Capelle. 


\section{La fonte nascosta: inventio e dissonanza}

\section{Silvia Ferrari}

Block, Lawrence. Ombre. Racconti ispirati ai dipinti di Edward Hopper. Trad. di Luca Briasco, Fabio Deotto e Letizia Sacchini, Einaudi, 2017.

Bonnefoy, Yves. Edward Hopper: la Photosynthèse de l'Être. Éditions Mercure de France, 1995.

—. Edward Hopper. La fotosintesi dell'essere. Trad. di Caterina Medici, Abscondita, 2009.

Conley, Katharine. Robert Desnos, Surrealism, and the Marvelous in Everyday Life. University of Nebraska, 2003.

Demont, François. "L'approche phénoménologique des figures de rhétorique chez Jean Paulhan." Exercices de rhétorique, vol. 15, 2020, journals.openedition.org/rhetorique/1062.

—_."Le dadaïsme et le surréalisme chez Jean Paulhan: pour une appréhension des discours et des dynamiques littéraires."Littérature, vol. 200, 2020, pp. 22-36.

Desnos, Robert. C'est les bottes des 7 lieues la phrase «je me vois». Éditions de la Galérie Simon, 1926.

—. Euvres. Gallimard, 1999.

_. Le vin est tiré... . Gallimard, 1943.

Dumas, Marie-Claire. Robert Desnos ou l'exploration des limites. Klincksieck,1980.

Egger, Anne. Robert Desnos. Fayard, 2007.

Frontisi, Claude. "Le mot « Klee »." Jeu surréaliste et humour noir, a cura Jacqueline ChénieuxGendron e Marie-Claire Dumas, Lachenal \& Ritter, 1993, pp. 219-36.

Ishikawa, Kiyoko. Paris dans quatre textes narratifs du surréalisme. Aragon, Breton, Desnos, Soupault. L'Harmattan, 1999.

Marmande, Francis. "Jazz et surréalisme: le jeu de l'instant." Jeu surréaliste et bumour noir, a cura di Chénieux-Gendron, Jacqueline e Marie-Claire Dumas, Lachenal \& Ritter, 1993, pp. 301-10.

Meschonnic, Henri. "Les Proverbes, actes du discours." Revue des sciences humaines, vol. XLI, 1976, pp. 419-30.

Ottinger, Didier. Hopper. Ombre et lumière du mythe américain. Gallimard, 2012.

Paulhan, Jean. Les fleurs de Tarbes ou la Terreur dans les Lettres. Gallimard, 1945.

—. Le guerrier appliqué. Gallimard, 1930.

—. Euvres complètes. Gallimard, 1945.

Renner, Rolf Günter. Hopper. 1991. Taschen, 2017.

Riese Hubert, Renée. "Proverbes et images dans Pénalités de l'enfer." «Moi qui suis Robert Desnos». Permanence d'une voix, a cura di Marie-Claire Dumas, José Corti, 1987, pp. 115-25.

Rossi Pinelli, Orietta. Hopper. Giunti, 2016.

Sounac, Frédéric. "Un paysage (dé)moralisé: trois regards contemporains sur Edward Hopper." Peinture et Littérature au XX'e siècle, a cura di Pascal Dethurens, Presses Universitaires de Strasbourg, 2007, pp. 437-48. 